University of Nebraska - Lincoln

DigitalCommons@University of Nebraska - Lincoln

$12-2019$

\title{
Lassa virus circulating in Liberia: a retrospective genomic characterisation
}

Michael R. Wiley

Lawrence S. Fakoli

Andrew G. Letizia

Stephen R. Welch

Jason T. Ladner

See next page for additional authors

Follow this and additional works at: https://digitalcommons.unl.edu/nsripubs

Part of the Defense and Security Studies Commons, Emergency and Disaster Management Commons, Other Public Affairs, Public Policy and Public Administration Commons, Science and Technology Policy Commons, and the Terrorism Studies Commons

This Article is brought to you for free and open access by the National Strategic Research Institute at the University of Nebraska at DigitalCommons@University of Nebraska - Lincoln. It has been accepted for inclusion in University of Nebraska's National Strategic Research Institute Publications by an authorized administrator of DigitalCommons@University of Nebraska - Lincoln. 


\section{Authors}

Michael R. Wiley, Lawrence S. Fakoli, Andrew G. Letizia, Stephen R. Welch, Jason T. Ladner, Karla Prieto, Daniel Reyes, Nicole Espy, Joseph A. Chitty, Catherine B. Pratt, Nicholas Di Paola, Fahn Taweh, Desmond Williams, Jon Saindon, William G. Davis, Ketan Patel, Mitchell Holland, Daniel Negrón, Ute Ströher, Stuart T. Nichol, Shanmuga Sozhamannan, Pierre E. Rollin, John Dogba, Tolbert Nyenswah, Fatorma Bolay, César G. Albariño, Mosoka Fallah, and Gustavo Palacios 


\title{
Lassa virus circulating in Liberia: a retrospective genomic characterisation
}

\author{
Michael R Wiley*, Lawrence Fakoli*, Andrew G Letizia*, Stephen R Welch*, Jason T Ladner*, Karla Prieto, Daniel Reyes, Nicole Espy, Joseph A Chitty, \\ Catherine B Pratt, Nicholas Di Paola, Fahn Taweh, Desmond Williams, Jon Saindon, William G Davis, Ketan Patel, Mitchell Holland, Daniel Negrón, \\ Ute Ströher, Stuart T Nichol, Shanmuga Sozhamannan, Pierre E Rollin, John Dogba, Tolbert Nyenswah, Fatorma Bolay, César G Albariño†, \\ Mosoka Fallaht, Gustavo Palacios $†$
}

\section{Summary}

Background An alarming rise in reported Lassa fever cases continues in west Africa. Liberia has the largest reported per capita incidence of Lassa fever cases in the region, but genomic information on the circulating strains is scarce. The aim of this study was to substantially increase the available pool of data to help foster the generation of targeted diagnostics and therapeutics.

Methods Clinical serum samples collected from 17 positive Lassa fever cases originating from Liberia (16 cases) and Guinea (one case) within the past decade were processed at the Liberian Institute for Biomedical Research using a targeted-enrichment sequencing approach, producing 17 near-complete genomes. An additional 17 Lassa virus sequences (two from Guinea, seven from Liberia, four from Nigeria, and four from Sierra Leone) were generated from viral stocks at the US Centers for Disease Control and Prevention (Atlanta, GA) from samples originating from the Mano River Union (Guinea, Liberia, and Sierra Leone) region and Nigeria. Sequences were compared with existing Lassa virus genomes and published Lassa virus assays.

Findings The 23 new Liberian Lassa virus genomes grouped within two clades (IV.A and IV.B) and were genetically divergent from those circulating elsewhere in west Africa. A time-calibrated phylogeographic analysis incorporating the new genomes suggests Liberia was the entry point of Lassa virus into the Mano River Union region and estimates the introduction to have occurred between 300-350 years ago. A high level of diversity exists between the Liberian Lassa virus genomes. Nucleotide percent difference between Liberian Lassa virus genomes ranged up to $27 \%$ in the $\mathrm{L}$ segment and $18 \%$ in the S segment. The commonly used Lassa Josiah-MGB assay was up to $25 \%$ divergent across the target sites when aligned to the Liberian Lassa virus genomes.

Interpretation The large amount of novel genomic diversity of Lassa virus observed in the Liberian cases emphasises the need to match deployed diagnostic capabilities with locally circulating strains and underscores the importance of evaluating cross-lineage protection in the development of vaccines and therapeutics.

Funding Defense Biological Product Assurance Office of the US Department of Defense and the Armed Forces Health Surveillance Branch and its Global Emerging Infections Surveillance and Response Section.

Copyright (C) 2019 Elsevier Ltd. All rights reserved.

\section{Introduction}

Lassa fever is a haemorrhagic disease caused by Lassa virus, a member of the Mammarenavirus genus in the family Arenaviridae. Lassa virus was first discovered in Nigeria in $1969^{1}$ and later detected in Liberia in $1972 .^{2}$ Historical evidence and molecular dating indicate that Lassa virus has circulated in west Africa for hundreds of years. ${ }^{3}$ The majority of the confirmed cases are from the Mano River Union region (Guinea, Liberia, and Sierra Leone) and Nigeria, but there is evidence of Lassa virus in Benin, Burkina Faso, Ghana, Côte d'Ivoire, Mali, and Togo. ${ }^{4}$

An estimated 300000 infections of Lassa fever occur annually, resulting in approximately 5000 deaths. ${ }^{4}$ For approximately $80 \%$ of these cases, symptoms are mild and undiagnosed. ${ }^{4}$ In the other $20 \%$ of infections, common symptoms include back and chest pain, facial

\section{U.S. government works are not subject to copyright.}

swelling, haemorrhaging, respiratory distress, and vomiting. ${ }^{4}$ The estimated fatality rate for all Lassa fever infections is $1 \%$, with a $15-20 \%$ case fatality rate in patients who are admitted to hospital. ${ }^{4}$ Because of the lack of standardised surveillance programmes, these numbers are all estimates and may not be an accurate representation of the true burden of Lassa fever in west Africa. Standardisation of surveillance has been complicated by the non-specific symptoms of Lassa fever, the high level of nucleotide (20-25\%) and amino acid (8-12\%) diversity within Lassa virus, and an overall lack of access to care for a large percentage of the population in endemic areas, resulting in a majority of clinical cases going undiagnosed. ${ }^{4-6}$

To address the lack of effective diagnostics, public and private groups have started addressing the gaps. Immunoassays have been developed to detect anti-Lassa

\section{CrossMark}

Lancet Infect Dis 2019; 19: $1371-78$

Published Online October 3, 2019 https://doi.org/10.1016/ S1473-3099(19)30486-4

See Comment page 1276

*Contributed equally

†Co-senior authors

Department of Environmental, Agricultural and Occupational Health, College of Public Health, University of Nebraska Medical Center, Omaha, NE, USA (M R Wiley PhD,

K Prieto MSc, D Reyes MSc (B Pratt MSc); Center for Genome Sciences, United States Army Medical Research Institute of Infectious Diseases, Frederick, MD, USA (M R Wiley, JT Ladner PhD, K Prieto, D Reyes, N Espy PhD, J A Chitty BSc, CB Pratt, N Di Paola ScD, G Palacios PhD); National Public Health Institute of Liberia, Monrovia, Liberia (L Fakoli BSC FTaweh MPH, J Dogba MPH, T Nyenswah MPH, F Bolay PhD M Fallah MD); Naval Medical Research Unit Three Ghana Detachment, Accra, Ghana (A G Letizia MD); US Centers for Disease Control and Prevention, Atlanta, GA, USA (SR Welch PhD, D Williams MD J Saindon PhD, W G Davis PhD, K Patel PhD, U Ströher PhD, ST Nichol PhD, PE Rollin MD, ( G Albariño PhD); US Centers for Disease Control and Prevention, Monrovia, Liberia (D Williams); Pathogen and Microbiome Institute, Northern Arizona University, Flagstaff, AZ, USA (JT Ladner); Noblis, Falls Church, VA, USA (M Holland MS, D Negrón MS); Defense Biological Product Assurance Office, Joint Program Executive Office for Chemical, Biological, Radiological and Nuclear Defense (CBRND)-Joint Project Lead, CBRND Enabling Biotechnologies, Frederick, MD, USA (S Sozhamannan PhD); 


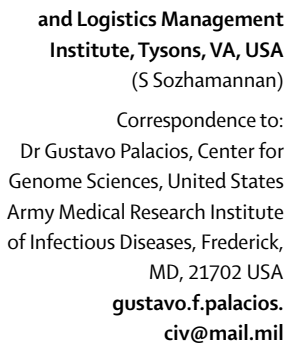

widerological and molecular diagnostics to capture the a give range of Lassa virus diversity that can be present in a given country. Reviews of currently available serological and molecular based diagnostics have been published. ${ }^{6,7}$

In 2018, Nigeria had an increase in Lassa fever cases associated with a case fatality rate of approximately $25 \%$, causing concern in the global public health community. ${ }^{9,10}$ Genomic analysis of the circulating strains compared with historical data indicated that the rise in cases was not caused by a new circulating variant or sustained human-tohuman transmission, but that it was consistent with transmission from the rodent reservoir., ${ }^{9,10}$ During the same period, Liberia also had a seasonal outbreak of Lassa fever. Despite the long history of Lassa virus in Liberia, ${ }^{2}$ a history of exported cases, ${ }^{11,12}$ and the high per capita rate of reported cases (table 1), little is known about the viral

\section{Added value of this study}

Liberia. This represents a ten-fold increase compared with the two coding-complete genomes currently found on GenBank. The new genomes span both temporally (1972-2017) and spatially (Bong, Nimba, Montserrado, and Lofa counties) across Liberia. Our data indicate that the Liberian Lassa virus strains are distinct from those circulating elsewhere and that improved molecular diagnostics are needed to detect Lassa fever more effectively in Liberia.

\section{Implications of all the available evidence}

The large genomic diversity of Lassa virus emphasises the need to match deployed diagnostics capabilities with locally circulating strains and underlines the importance of evaluating cross-lineage protection in the development of vaccines and therapeutics. The data also highlight the need to increase Lassa fever surveillance using appropriate diagnostics, and to implement strategies that can cope with high levels of diversity. genetic diversity circulating in this country. We report 17 Lassa virus genome sequences from clinical cases (one from Guinea and 16 from Liberia) and 17 from viral stocks (two from Guinea, seven from Liberia, four from Nigeria, and four from Sierra Leone). We used these genomes to infer the evolutionary history of Lassa virus in Liberia and more broadly in west Africa, and investigated the appropriateness of current sequence-based diagnostic tools for detecting Lassa virus lineages from Liberia.

\section{Methods}

\section{Sample processing and sequencing}

De-identified human serum samples obtained from patients determined to be positive for Lassa fever by the Liberian National Reference Laboratory were processed for sequencing at the Liberian Institute for Biomedical Research. Accompanying metadata were provided by the National Public Health Institute of Liberia (ethics statement is provided in the appendix, p 3). Samples in Liberia were sequenced on an Illumina MiSeq at the Liberian Institute for Biomedical Research using an enrichment-based method as previously described ${ }^{13}$ with modifications to enrich Lassa virus. Archival isolates at the US Center for Disease Control and Prevention (CDC) Viral Special Pathogens branch in Atlanta, GA, were sequenced using the TruSeq Stranded mRNA kit on an Illumina MiniSeq. Additional details on sequencing methods can be found in the appendix (p 3). The consensus genome sequences were deposited in GenBank (appendix p 6).

\section{Phylogenetics and molecular clock analysis}

We generated Large (L) and Small (S) segment-level coding sequence alignments (concatenated open reading frames) 


\begin{tabular}{|c|c|c|c|c|c|}
\hline & 2014 & 2015 & 2016 & 2017 & 2018 \\
\hline \multicolumn{6}{|l|}{ Liberia } \\
\hline Reported cases & 10 & 3 & 96 & 81 & 191 \\
\hline Confirmed cases & 1 & 0 & 14 & 30 & 21 \\
\hline Confirmation rate & $10 \cdot 0 \%$ & 0 & $14 \cdot 6 \%$ & $37 \cdot 0 \%$ & $11.0 \%$ \\
\hline Population size (million) & $4 \cdot 39$ & $4 \cdot 50$ & $4 \cdot 61$ & 4.73 & 4.85 \\
\hline Reported per 100000 & 0.23 & 0.07 & $2 \cdot 08$ & 1.71 & 3.94 \\
\hline Confirmed per 100000 & 0.02 & 0.00 & $0 \cdot 30$ & 0.63 & 0.43 \\
\hline \multicolumn{6}{|l|}{ Nigeria } \\
\hline Reported cases & 929 & 430 & 901 & 1022 & 3276 \\
\hline Confirmed cases & 110 & 25 & 101 & 308 & 588 \\
\hline Confirmation rate & $11 \cdot 8 \%$ & $5 \cdot 8 \%$ & $11 \cdot 2 \%$ & $30 \cdot 1 \%$ & $17 \cdot 9 \%$ \\
\hline Population size (million) & $176 \cdot 46$ & $181 \cdot 18$ & 185.99 & $190 \cdot 87$ & $195 \cdot 88$ \\
\hline Reported per 100000 & 0.53 & 0.24 & 0.48 & 0.54 & 1.67 \\
\hline Confirmed per 100000 & 0.06 & 0.01 & 0.05 & 0.16 & $0 \cdot 30$ \\
\hline
\end{tabular}

in Geneious (version 11.1.4; Biomatters, Auckland, New Zealand) using publicly available sequences covering at least $80 \%$ of the protein-coding portions of either the L or S genome segment. Maximum-likelihood phylogenies were generated using RAxML Next Generation with the GTR+G4 model and 100 bootstrap replicates. ${ }^{14}$ Root-to-tip distances using the same dataset were generated with TempEst (v1.5.1) ${ }^{15}$ using the best-fitting root option. Bayesian phylogenies for each of the segment-level alignments were generated in BEAST (v1.8.4). ${ }^{16}$ Detailed methods for the analysis and model comparisons are provided in the appendix (pp 3-4, p 7).

\section{Lassa virus PCR assay evaluation}

Lassa virus specific PCR assays were evaluated using both in vitro and in silico methods. The in vitro analysis was done using the two assays deployed in Liberia, the Nikisins $^{17}$ and Trombley Josiah-MGB. ${ }^{18}$ The in silico analysis was done with the PCR Signature Erosion Tool (version 1$)^{19}$ using 16 published Lassa virus assays, including the Nikisins and Trombley Josiah-MGB. A final in silico analysis was done by aligning the PCR primers and probes to all the available Lassa virus sequences and measuring the number of mismatches (appendix p 8, pp 12-21, p 24). A more detailed description of the assay evaluation can be found in the appendix (p 4).

\section{Role of the funding source}

The sponsor of the study had no role in study design, data collection, data analysis, data interpretation, or writing of the report. The corresponding author had full access to all the data in the study and had final responsibility for the decision to submit for publication.

\section{Results}

Serum samples positive for Lassa virus were provided by the National Public Health Institute of Liberia and were processed and sequenced at the Liberian Institute for Biomedical Research. We sequenced six samples from 2018 (one was an imported case from Guinea) and 11 archival samples collected between 2011 and 2017 (table 2). Most samples originated from known Lassa fever hotspots in Bong and Nimba counties. Cases from Montserrado county probably represent imported cases from endemic counties-for instance, the patient of sample LIB-LF17-001 was a resident of Ganta in Nimba county who became febrile in Montserrado. Codingcomplete genomes were obtained for 12 samples; the remaining five had gaps in coverage in the $\mathrm{L}$ protein (appendix p 6). We generated 17 coding-complete sequences from viral stocks of historical reference samples (two from Guinea, seven from Liberia, four from Nigeria, and four from Sierra Leone), from the Centers for Disease Control Viral Special Pathogens Branch (CDC-VSPB) as well. Of the seven samples originating from Liberia, three were imported into the USA with clear epidemiological links to Liberia. . $^{11,12}$ Pairwise comparisons of the new coding-complete Liberian genomes showed nucleotide diversity that ranged up to $27 \%$ in the $\mathrm{L}$ segment and $18 \%$ in the $\mathrm{S}$ segment.

Segment-level phylogenetic analysis, including publicly available Lassa virus genomes, indicated that all of our Liberian genomes belong to lineage IV (figure 1)..$^{20,21}$ The only exception was the L segment from isolate 807978-P28, which falls outside of the diversity previously characterised for lineage IV (appendix p 22). The Liberian Lassa virus genomes are split between two major clades within lineage IV (labelled IV.A and IV.B in the
For the Liberia IDSR Epidemiology Bulletin see https://nationalphil.org For more on Worldometers see http://www.worldometers.info/ world-population 


\begin{tabular}{|c|c|c|c|c|c|c|}
\hline & Country & County or prefecture & District of residence & Health-care facility & Specimen type & Collection date \\
\hline LIB-LF11-003 & Liberia & Bong & Suakoko & Phebe Hospital & Serum & Feb 20, 2011 \\
\hline LIB-LF11-011 & Liberia & Bong & Jorkoqulleh & Phebe Hospital & Serum & April 14, 2011 \\
\hline LIB-LF13-006 & Liberia & Nimba & Seclepea Mah & Seclepea Health Center & Serum & April 29, 2013 \\
\hline LIB-LF13-007 & Liberia & Nimba & Seclepea Mah & Seclepea Health Center & Serum & April 29, 2013 \\
\hline LIB-LF16-004 & Liberia & Bong & Jorkoqulleh & Phebe Hospital & Serum & Feb 26, 2016 \\
\hline LIB-LF16-016 & Liberia & Nimba & Garr Bain & Ganta United Methodist Hospital & Serum & April 13, 2016 \\
\hline LIB-LF17-001 & Liberia & Montserrado* & Ganta & Ganta United Methodist Hospital & Serum & Jan 9, 2017 \\
\hline LIB-LF17-007 & Liberia & Nimba & Seclepea Mah & Ganta United Methodist Hospital & Serum & Jan 9, 2017 \\
\hline LIB-LF17-013 & Liberia & Bong & Jorkoqulleh & Phebe Hospital & Serum & Feb 13, 2017 \\
\hline LIB-LF17-019 & Liberia & Bong & Jorkoqulleh & Phebe Hospital & Serum & May 4, 2017 \\
\hline LIB-LF17-020 & Liberia & Nimba & Seclepea Mah & Phebe Hospital & Serum & May 2, 2017 \\
\hline LIB-LF18-001 & Liberia & Bong & NA & NA & Serum & Jan 10, 2018 \\
\hline LIB-LF18-002 & Guinea & Yomou & NA & Diécké & Serum & Jan 10, 2018 \\
\hline LIB-LF18-037 & Liberia & Nimba & NA & NA & Serum & Feb 13, 2018 \\
\hline LIB-LF18-040 & Liberia & Montserrado & NA & NA & Serum & March 2, 2018 \\
\hline LIB-LF18-041 & Liberia & Montserrado & NA & NA & Serum & March 3, 2018 \\
\hline LIB-LF18-042 & Liberia & Nimba & NA & NA & Serum & March 1, 2018 \\
\hline \multicolumn{7}{|c|}{$\begin{array}{l}\text { Samples were provided by the National Public Health Institute of Liberia and were processed and sequenced at the Liberian Institute for Biomedical Research. Country, } \\
\text { county, and prefecture indicate where the patient first presented with symptoms and district of residence indicates where the patient lives. NA=not available. } \\
\text { *Patient was in Montserrado county when becoming febrile but suspected to have become infected in Nimba county. }\end{array}$} \\
\hline
\end{tabular}

$\mathrm{L}$ and $\mathrm{S}$ genome segment trees of figures 1 and 2 ). The genome segments from the 2018 imported case from Guinea grouped into clade IV.A which, until now, only contained partial S genome segments of Liberian origin (appendix p 23). Clade IV.B had been observed in Guinea, Liberia, and Sierra Leone and contains the two previously available coding-complete genomes from Liberia. We estimated that these two clades diverged approximately 300-350 years ago (L: median 1669, 95\% highest posterior density [HPD] interval 1616-1717]; S: median 1720, 95\% HPD interval 1674-1758) by fitting a molecular clock to the data. Notably, the Liberian isolate 807978-P28 appears to be a reassortant, with an S segment from clade IV.A and an L segment that does not fall within lineage IV or $\mathrm{V}$ but belongs to a previously unobserved phylogenetic lineage (appendix pp 22-23). A more detailed analysis below the lineage level is provided in the appendix (pp 4-5).

Our molecular clock analysis of lineages IV and $\mathrm{V}$ estimated substitution rates (L: $8.4 \times 10^{-4}$ substitutions per site per year, $95 \%$ HPD interval $7.6 \times 10^{-4}$ to $9.2 \times 10^{-4}$; S: $8 \cdot 2 \times 10^{-4}, 95 \%$ HPD interval $7 \cdot 2 \times 10^{-4}$ to $9 \cdot 1 \times 10^{-4}$ ) that are consistent with previous studies ${ }^{3,21}$ that included all five Lassa virus lineages. Our estimates for the time of most recent common ancestors (tMRCA) for the large clade from Sierra Leone in lineage IV and lineage $\mathrm{V}$ (figure 2A and $2 \mathrm{~B}$ ) are in line with previous estimates of about 150 years ago.,21 However, new genomes from Liberia and Guinea have pushed back estimates for the introduction of Lassa virus into the Mano River Union from about 220 to $300-350$ years ago (figure 2A and 2B). ${ }^{3}$ Both S-based and L-based segment estimates were highly consistent. Our analysis indicated that with the current data, Liberia was most likely the entry point for Lassa virus to the Mano River Union (posterior probability $\geq 94 \%$ ), and that the virus was later introduced, at least four times, into Guinea $(0-40,40-60,50-130$, and 90-280 years ago) and at least once into Sierra Leone (about 150 years ago; figure 2A and 2B). Root-to-tip distances were also generated using the identical dataset and a positive correlation between sampling date and root-to-tip distance is observed, indicative of molecular clock signal in the data (figure 2C and 2D).

Liberia was identified as the most likely location for the MRCA of lineages IV and V, although the low posterior support indicates ambiguity in this assignment with the current dataset. Although the $\mathrm{S}$ and L segment trees were highly consistent, they differed substantially in their tMRCA estimates for the ancestor of lineages IV and V. This is likely due to the L segment of isolate 807978-P28, which, as mentioned previously, is consistent with a reassortment event and does not belong to either lineage IV or V. Evidence for an additional sequence outside of lineage IV and $\mathrm{V}$ is present in the $\mathrm{S}$ genome segment phylogeny after inclusion of the partial genomes (appendix p 23). These results suggest that the genetic diversity of Lassa virus remains undersampled. Thus, these tMRCAs will likely need further revision with the addition of more sequence data.

The Liberian National Reference Laboratory in Charlesville uses two quantitative real-time PCRs (rtPCRs) assays to detect Lassa virus: the Lassa Josiah-MGB assay (which was designed to detect the lineage IV and targets the glycoprotein precursor [GPC] gene on the $\mathrm{S}$ genome 


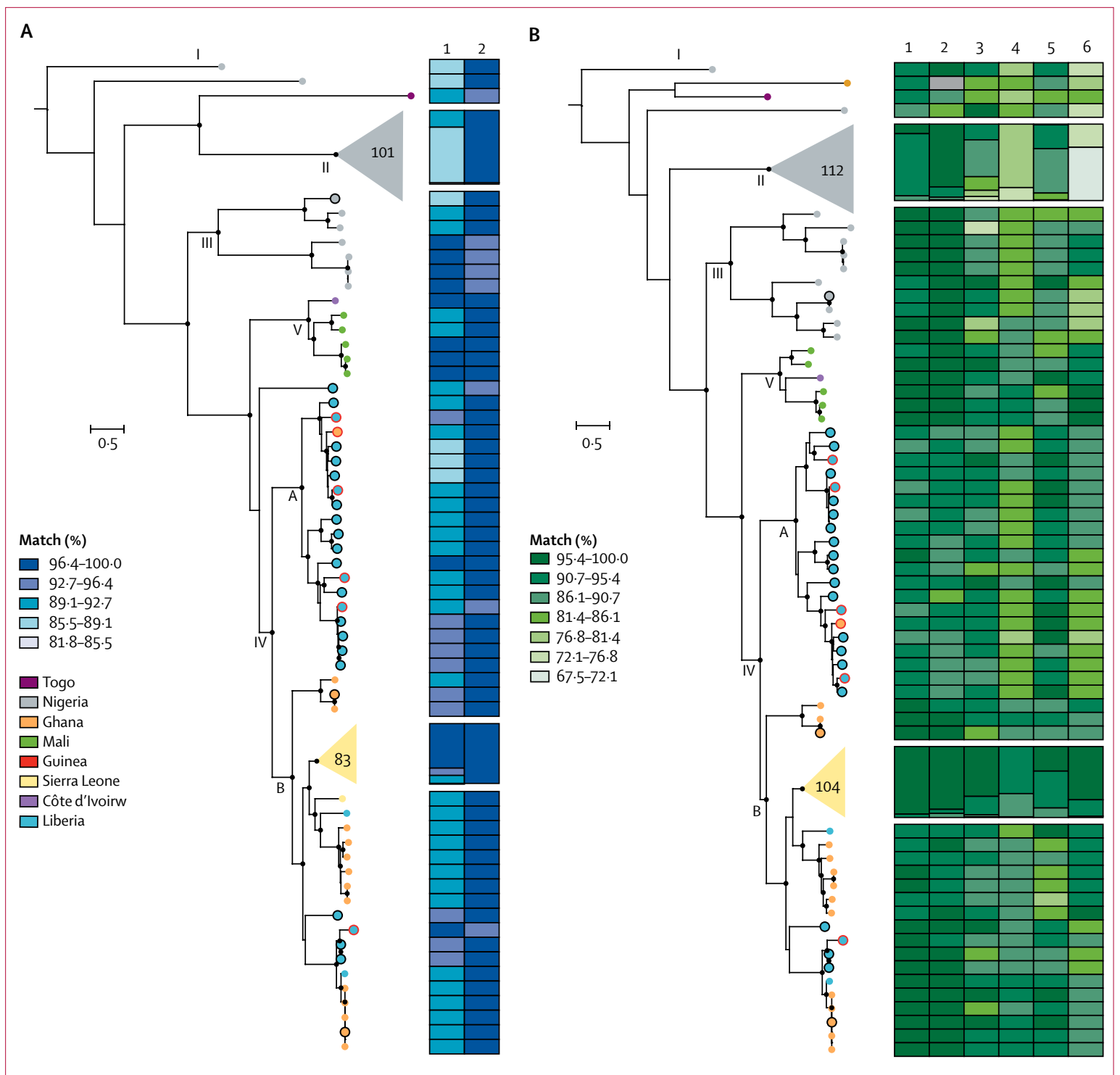

Figure 1: Maximum-likelihood trees for Lassa virus $L$ and $S$ genome segments sequenced from human infections and reservoir hosts in west Africa (A) Maximum-likelihood tree for $\mathrm{L}$ genome segment. (B) Maximum-likelihood tree for S genome segment. Nodes are coloured based on the country of origin. Sequences generated from the 2018 cases are outlined in red (see table 2 and appendix $\mathrm{p} 6$ for sample metadata and sequence completeness), other sequences from this study are outlined in black. For the collapsed clades, the number of contained sequences is specified. Scale bars indicate number of nucleotide substitutions per site. Roman numerals are used to label previously defined Lassa virus lineages ${ }^{20,21}$ (see appendix pp 22-23 for subtrees of lineage IV and $V$ which include partial sequences). The heatmaps to the right of the phylogenetic trees in panels A and B represent the percent identity of the assay target site as presented in the appendix (pp 12-21). The numbers above the heatmap in panel $\mathrm{A}$ represent the following assays: 1 Nikisins ${ }^{22}$ and 2 Vieth. ${ }^{17}$ The numbers above the heatmap in panel $\mathrm{B}$ represent the following assays: 1 Bowen, ${ }^{20} 2$ Coulibaly, ${ }_{r}^{23} 3$ Demby, ${ }_{r}^{24} 4$ Leski, $^{25} 5$ Olschläger $_{,}{ }^{26}$ and 6 Safronetz $^{27}$ (see appendix $\mathrm{p} 8$ for assay sequences and the gene targets).

segment) ${ }^{18}$ and the La-V assay (designated Nikisins, which was designed to be a pan-Lassa assay and targets the RNAdependent RNA polymerase [L] gene on the $\mathrm{L}$ genome segment). ${ }^{17}$ Samples analysed at the Liberian National Reference Laboratory revealed a large number of discrepancies between the results of the two assays; the Nikisins assay often produced positive results for samples for which the Lassa Josiah-MGB was negative. We aligned the primers and probes from the two assays to the newly sequenced Lassa virus genomes and observed a large number of mismatches in the target sites of the Lassa Josiah-MGB assay (75 · 0-98 .4\% identity across target sites, as seen in appendix p 24). Fewer mismatches were present in the targeted regions of the Nikisins assay (94.6-100\% identity across target sites), mainly due to well-placed ambiguous bases to account for Lassa virus diversity.

To determine how these mismatches would affect the ability to detect Lassa virus using the Nikisins or Lassa Josiah-MGB assays, we did in vitro tests using 16 viral stocks that originated from west Africa (appendix $\mathrm{p} 9$ ). 
Figure 2: Temporal maximum clade credibility trees for Lassa virus $L$ and $S$ genome segments sequenced from human infections and reservoir hosts in west Africa (A) Temporal maximum clade credibility tree for $\mathrm{L}$ genome segment. (B) Temporal maximum clade credibility tree for $S$ genome segment. Only lineages IV and V were included in this analysis.

Small black circles indicate clades with posterior probabilities of at least $90 \%$. Branches are coloured according to the inferred ancestral location of each lineage. Coloured rings indicate nodes with ancestral state posterior probabilities of at least $90 \%$; nodes below this threshold are labelled with their respective probabilities. The grey violin plots represent the $95 \%$ highest posterior density intervals for the time of most recent common ancestor (tMRCA) for clades IV.A, IV.B, lineage IV, and lineages IV and $V$ together (tree root). Liberian samples with known county of origin are indicated with filled circles and coloured according to county of origin. Since the L genome segment of sample 807978-P28 fell outside of the diversity previous described for lineages IV it was not used in the TMRCA analysis for this lineage. (C) Root-to-tip distances for the $L$ genome segment. (D) Root-to-tip distances for the $\mathrm{S}$ genome segment. Colours indicate country of origin and the line represents a simple linear regression (see appendix $\mathrm{p} 7$ for the BEAST model comparison for the $L$ and S genome segments).
Four ten-fold dilutions of each viral stock were tested to see if either assay could detect all the dilutions. The Nikisins assay was able to detect all the Liberian isolates at every dilution; however, the Josiah-MGB assay was only able to detect two of the seven Liberian isolates at all four dilutions. A correlation was observed between the

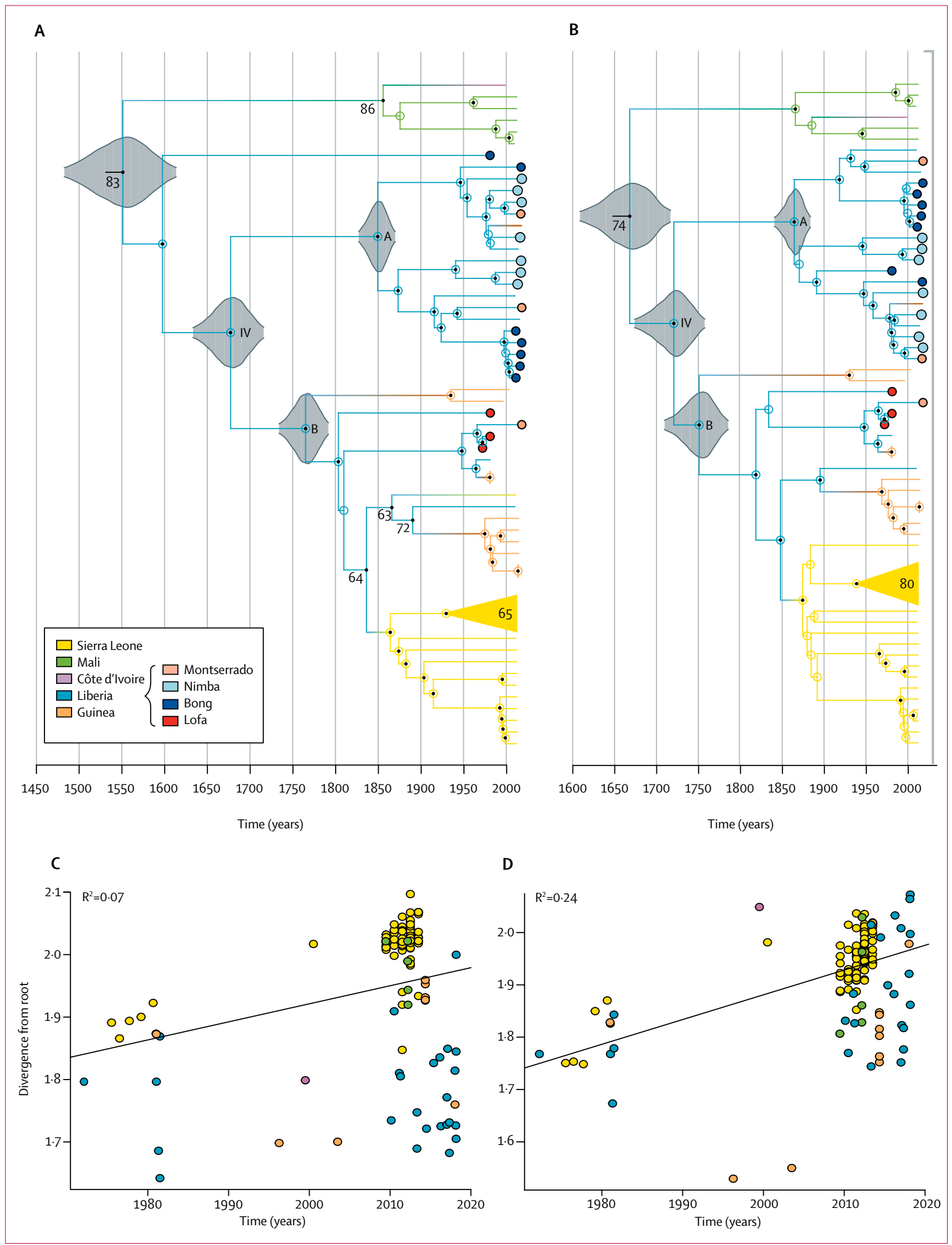


number of mismatches in the target region and ability to detect all dilutions.

Because of the discrepancies observed between the Lassa Josiah-MGB assay and the Nikisins assay, we expanded our evaluation to other published Lassa virus rtPCR assays using in silico methods. The goal was to quickly evaluate alternatives for detection of Lassa virus in Liberia and other endemic regions in Africa. We evaluated 16 published assays with the PCR Signature Erosion Tool (appendix p 10) by calculating the percent identity across the target regions and inferring the minimal number of assays needed to cover all the diversity in the L and S segment (appendix p 11). We listed the percent identities of the two L segment assays and the top six S segment assays along with accession numbers (appendix pp 12-22) and presented the data as a heatmap in figure 1 to coordinate with the tips of the phylogenetic tree. Among the two published L segment assays tested, the Nikisins assay showed the highest percent identity across the target site of the analysed Lassa virus genomes, with a range of $94 \cdot 6-100 \%$ and an average of $98.6 \%$ (figure 1; appendix pp 12-22). Between the six $\mathrm{S}$ segment assays tested, the Bowen ${ }^{20}$ and Coulibaly ${ }^{23}$ assays had the highest average identity at $95 \cdot 2 \%$ for Bowen and $96.1 \%$ for Coulibaly (appendix pp 12-22). When focusing on just genomes of Liberian origin, the Ölschläger assay $^{26}$ had the highest average identity for an S segment assay at $94 \cdot 2 \%$.

\section{Discussion}

Liberian Lassa virus genomes are substantially underrepresented in genomic databases by comparison with other Mano River Union countries and Nigeria. As of January, 2019, only two coding-complete Lassa virus genomes, 36 partial $\mathrm{S}$ genome segments (27 GPC at $60-80 \%$ coverage and nine nucleoprotein at $40 \%$ coverage), and six partial L genome segments (6-12\% coverage of L gene) of Liberian origin were available on GenBank. The short sequence length limits the type of downstream analyses that can be done compared with coding-complete genomes. To address the scarcity of genetic information, we obtained coding-complete viral genomes from confirmed cases during the 2018 Lassa fever season and from a collection of archival samples. In total, we were able to generate 34 near-complete genomes for the L and S segments, 23 of these coming from samples of Liberian origin (figure 1; table 2; appendix p 6).

These newly sequenced genomes show a large amount of diversity is present among circulating strains in Liberia. The genomes were grouped into two distinct and wellsupported clades, which we designated IV.A and IV.B. When geographical information was available, we found that the Liberian genomes grouped according to location rather than collection dates, which is consistent with previous observations in Guinea, Nigeria, and Sierra Leone. ${ }^{3,9,10,20,25,28}$ Most newly sequenced samples grouped in clade IV.A, which until now had only been represented in GenBank by partial genomes (figure 1; appendix pp 22-23).
The Liberian genomes from clade IV.A originate from Bong, Montserrado, and Nimba counties, and could not be resolved further than county-level based on the available metadata. Clade IV.A could be split further into three well supported subclades that include a grouping of all three counties, a group of Bong and Montserrado counties, and a group of just Nimba county. The imported 2018 case from Guinea also grouped in clade IV.A, specifically in the subclade that included representatives from Bong, Montserrado, and Nimba. More details on the movement of Lassa fever between Liberia and Guinea can be found in the appendix (p 5).

Clade IV.B has been previously observed in Guinea, Liberia, and Sierra Leone, and both of the previously available coding-complete genomes from Liberia belong to this clade. The majority of Liberian genomes that belong to this clade are older (originating from 1972-81) and were isolated from patients diagnosed at Curran Lutheran Hospital in Zorzor, Liberia, in Lofa County. ${ }^{29,30}$ One of the 2018 samples, LF18040, falls within a well-supported subclade of IV.B, which had not been detected for the past 37 years (appendix pp 22-23), showing that this clade continues to circulate.

Even with additional sequencing data, it appears further sampling is still needed to capture all the Lassa virus diversity circulating in Liberia. Multiple indicators of this include the $\mathrm{L}$ and $\mathrm{S}$ genome segments of the previously reported G1200 strain (KM821797 and KM821798), which group only with sequences from Guinea and Sierra Leone, and the L segment of strain 807978-P28 and the partial $\mathrm{S}$ segment from the previously reported Lib05-4094 strain (GU830819), which both fall outside of lineage IV and V (appendix pp 22-23). The available metadata indicate that 807978-P28 is from Bong country, but for G1200 and Lib05-4094 only the country of origin is provided, making it difficult to determine the region of the country in which this diversity is present. Additional Lassa virus surveillance and sequencing of confirmed cases is required to ensure that the complete viral diversity in Liberia has been covered.

To expand successful surveillance activities of Lassa fever in Liberia, deployed diagnostics need to detect all of the known genomic diversity. Reliance solely on sequencebased assays can cause limitations as shown in a 2015 international quality assessment study. ${ }^{17}$ In this study, Nikisins and colleagues ${ }^{17}$ observed 11 out of 24 international reference laboratories had at least one false-negative result and four of these laboratories did not identify any of the samples originating from Liberia. ${ }^{17}$ One solution would be to switch to multiple pan-Lassa assays that target the $\mathrm{L}$ and S segment (eg, Altona RealStar Nikisins assays), as done during the 2018 Lassa fever outbreak in Nigeria to help eliminate false-negative results due to potential target erosion. ${ }^{10}$ Another solution would be to incorporate immunoassays into the testing algorithms as has been shown in field testing in Sierra Leone. ${ }^{8}$ The rapid diagnostic antigen test was able to detect samples missed by the
For more on the Altona kits see https://www.altona-diagnostics. com/en/products/reagents-140/ reagents/realstar-real-time-pcrreagents/realstar-lassavirus-rtpcr-kit-ruo.html 
rtPCR assay and new pan-Lassa rapid diagnostic tests could provide an even broader detection capacity.

The dramatic increase of Lassa fever cases in west Africa, including Liberia and Nigeria, highlights the growing risk Lassa virus poses to regional and global health security. The overwhelming amount of observed Lassa virus diversity underscores the requirement that medical countermeasures and diagnostic assays developed against commonly used Lassa virus laboratory strains are reevaluated locally to ensure effectiveness at preventing, detecting, and treating all the circulating strains. Our data identify concerns in the current molecular diagnostic assays being used in Liberia and highlight the need to evaluate more recently developed pan-Lassa rtPCR assays and rapid diagnostic tests in Liberia, to develop a more comprehensive testing algorithm. Our results are probably not unique to Liberia and emphasise that these problems might not be completely resolved if we focus too much on any given country or region.

\section{Contributors}

MRW, AGL, CGA and GP conceptualized the study. MRW, JTL, NE, ND, JAC, HM, DN and SS analysed the data. MRW, LF, SW, KaPr, DR, WGD, and KePa generated the data. FT, JD, TN and MF provided study resources. MRW, AGL, JTL, CBP, SS, PER, CGA and GP wrote and edited the manuscript. DW, JS, US, STN, TN, FB, CGA, MF, and GP supervised the study.

Declaration of interests

We declare no competing interests.

\section{Data sharing}

Lassa virus consensus genomes are available on GenBank under accession numbers MG812630-MG812685 and MH215278-MH215289.

\section{Acknowledgments}

The views expressed in this article are those of the authors and do not necessarily reflect the official policy or position of the Department of the Army, the Department of the Navy, the Department of Defense, the US Department of Health and Human Services, the US Government, nor the institutions or companies affiliated with the authors. Financial support for this work was provided by the Defense Biological Product Assurance Office through task order awarded to the National Strategic Research Institute under FA4600-12-D-9000, the Armed Forces Health Surveillance Branch and its Global Emerging Infections Surveillance and Response (GEIS) Section (funding year 2017, ProMIS ID P2052_16_N3), and GEIS outbreak funds through Navy Medical Research Unit Three Ghana Detachment. CDR AGL is a military service member of the US Government. This work was prepared as part of his official duties. Title 17 of the United States Code $\$ 105$ provides that “Copyright protection under this title is not available for any work of the United States Government." Title 17 of the United States Code $\$ 101$ defines a US Government work as a work prepared by a military service member or employee of the US Government as part of that person's official duties. JT Ladner was funded under the State of Arizona Technology and Research Initiative Fund (TRIF), administered by the Arizona Board of Regents, through Northern Arizona University.

References

1 Frame JD, Baldwin JM Jr, Gocke DJ, Troup JM. Lassa fever, a new virus disease of man from west Africa. I. Clinical description and pathological findings. Am J Trop Med Hyg 1970; 19: 670-76.

2 Monath TP, Mertens PE, Patton R, et al. A hospital epidemic of Lassa fever in Zorzor, Liberia, March-April 1972. Am J Trop Med Hyg 1973; 22: 773-79.

3 Andersen KG, Shapiro BJ, Matranga CB, et al. Clinical sequencing uncovers origins and evolution of Lassa virus. Cell 2015; 162: 738-50.

4 Centers for Disease Control and Prevention. Lassa fever. Jan 31, 2019. https://www.cdc.gov/vhf/lassa/index.html (accessed June 26, 2019).
5 Gibb R, Moses LM, Redding DW, Jones KE. Understanding the cryptic nature of Lassa fever in west Africa. Pathog Glob Health 2017; 111: $276-88$.

6 Mazzola L, Kelly-Cirino C. Diagnostics for Lassa fever virus: a genetically diverse pathogen found in low-resource settings. BMJ Global Health 2019; 4 (suppl 2): e001116.

7 Raabe V, Koehler J. Laboratory diagnosis of Lassa fever J Clin Microbiol 2017; 55: 1629-37.

8 Boisen ML, Hartnett JN, Shaffer JG, et al. Field validation of recombinant antigen immunoassays for diagnosis of Lassa fever. Sci Rep 2018; 8: 5939.

9 Siddle KJ, Eromon P, Barnes KG, et al. Genomic analysis of Lassa virus during an increase in cases in Nigeria in 2018. N Engl J Med 2018; 379: 1745-53.

10 Kafetzopoulou LE, Pullan ST, Lemey P, et al. Metagenomic sequencing at the epicenter of the Nigeria 2018 Lassa fever outbreak. Science 2019; 363: 74-77.

11 Kulkarni PA, Chew D, Youssef-Bessler M, et al. Case report: imported case of fever-New Jersey, May 2015. Am J Trop Med Hyg 2018; 99: 1062-65.

12 Choi MJ, Worku S, Knust B, et al. A case of Lassa fever diagnosed at a community Hospital-Minnesota 2014. Open Forum Infect Dis 2018. 5: ofy131.

13 Mate SE, Kugelman JR, Nyenswah TG, et al. Molecular evidence of sexual transmission of Ebola virus. N Engl J Med 2015; 373: 2448-54.

14 Stamatakis A. RAxML version 8: a tool for phylogenetic analysis and post-analysis of large phylogenies. Bioinformatics 2014; 30: 1312-13.

15 Rambaut A, Lam TT, Max Carvalho L, Pybus OG. Exploring the temporal structure of heterochronous sequences using TempEst (formerly Path-O-Gen). Virus Evol 2016; 2: vew007.

16 Drummond AJ, Suchard MA, Xie D, Rambaut A. Bayesian phylogenetics with BEAUti and the BEAST 1.7. Mol Biol Evol 2012; 29: 1969-73.

17 Nikisins S, Rieger T, Patel P, Müller R, Günther S, Niedrig M. International external quality assessment study for molecular detection of Lassa virus. PLoS Negl Trop Dis 2015; 9: e0003793.

18 Trombley AR, Wachter L, Garrison J, et al. Comprehensive panel of real-time TaqMan polymerase chain reaction assays for detection and absolute quantification of filoviruses, arenaviruses, and New World hantaviruses. Am J Trop Med Hyg 2010; 82: 954-60.

19 Sozhamannan S, Holland MY, Hall AT, et al. Evaluation of signature erosion in Ebola virus due to genomic drift and its impact on the performance of diagnostic assays. Viruses 2015; 7: 3130-54.

20 Bowen MD, Rollin PE, Ksiazek TG, et al. Genetic diversity among Lassa virus strains. J Virol 2000; 74: 6992-7004.

21 Manning JT, Forrester N, Paessler S. Lassa virus isolates from Mali and the Ivory Coast represent an emerging fifth lineage. Front Microbiol 2015; 6: 1037.

22 Vieth S, Drosten C, Lenz O, et al. RT-PCR assay for detection of Lassa virus and related Old World arenaviruses targeting the $\mathrm{L}$ gene. Trans R Soc Trop Med Hyg 2007; 101: 1253-64.

23 Coulibaly-N'Golo D, Allali B, Kouassi SK, et al. Novel arenavirus sequences in Hylomyscus sp. and Mus (Nannomys) setulosus from Cote d'Ivoire: implications for evolution of arenaviruses in Africa. PLoS One 2011; 6: e20893.

24 Demby AH, Chamberlain J, Brown DW, Clegg CS. Early diagnosis of Lassa fever by reverse transcription-PCR. J Clin Microbiol 1994; 32: 2898-903.

25 Leski TA, Stockelman MG, Moses LM, et al. Sequence variability and geographic distribution of Lassa virus, Sierra Leone. Emerg Infect Dis 2015; 21: 609-18.

26 Olschläger S, Lelke M, Emmerich P, et al. Improved detection of Lassa virus by reverse transcription-PCR targeting the 5' region of S RNA. J Clin Microbiol 2010; 48: 2009-13.

27 Safronetz D, Lopez JE, Sogoba N, et al. Detection of Lassa virus, Mali. Emerg Infect Dis 2010; 16: 1123-26.

28 Fichet-Calvet E, Ölschläger S, Strecker T, et al. Spatial and tempora evolution of Lassa virus in the natural host population in Upper Guinea. Sci Rep 2016; 6: 21977.

29 Frame JD. Clinical features of Lassa fever in Liberia. Clin Infect Dis 1989; 11 (suppl 4): S783-89.

30 Jahrling PB, Frame JD, Smith SB, Monson MH. Endemic Lassa fever in Liberia. III. Characterization of Lassa virus isolates. Trans R Soc Trop Med Hyg 1985; 79: 374-79. 